\title{
Campylobacter Bacteremia in Hemodialysis Patients by Eating Raw Meat - The Importance of Sanitary Education
}

\author{
Yoshio Shimizu Arisa Ishii Akiko Takahata \\ Tadahiro Kajiyama Aya Yamahatsu Hiroaki lo \\ Atsushi Kurusu Chieko Hamada Satoshi Horikoshi \\ Yasuhiko Tomino
}

Division of Nephrology, Department of Internal Medicine, Juntendo University

Faculty of Medicine, Tokyo, Japan

\author{
Key Words \\ Campylobacter · Hemodialysis · Sanitary education
}

\begin{abstract}
In 2011, simultaneous, widespread outbreaks of food poisoning by contaminated enterohemorrhagic Escherichia coli in beef, which killed four and hospitalized more than 30 people, occurred in Japan. While the press was widely reporting this disaster, two maintenance hemodialysis patients were suffering from Campylobacter bacteremia by eating undercooked meat. One patient was infected with $C$. upsaliensis and the other with C. fetus. Although these patients could be successfully treated, they led us to consider the characteristics of $C$. upsaliensis and $C$. fetus as opportunistic pathogens, as well as changes in dietary behaviors and food markets. Moreover, they emphasized the need for hemodialysis patients to be not only educated in that they should restrict potassium, phosphate and water intake, but also that they should take care of food sanitation.
\end{abstract}

\section{Introduction}

Campylobacter bacteria are motile, non-spore-forming, comma-shaped, Gramnegative rods [1]. Within the Campylobacter species, $C$. jejuni and $C$. coli are reported as the most common cause of human acute bacterial enterocolitis. However, other atypical 
and emerging Campylobacter species, including C. upsaliensis and C. fetus, have been identified as pathogens with various clinical manifestations [2]. They are commonly observed as commensals of the gastrointestinal tracts of wild or domesticated cattle, sheep, goats, swine, dogs, cats and other exotic pets [3]. Sporadic cases of human infection are generally attributed to the consumption of raw or undercooked poultry meat [4], though outbreaks have been traced to the contamination of the water supply and the consumption of contaminated raw milk [5].

In 2011, four people died and more than thirty-five people were hospitalized due to fatal food poisoning caused by raw beef dishes served at a yakiniku barbecue restaurant chain in Toyama and Kanagawa prefectures in Japan [6]. In many of these cases, enterohemorrhagic E. coli, including 0-111 and 0-157 strains, were detected. In order to prevent another outbreak, the Ministry of Health, Labor and Welfare of Japan tightened regulations on food safety [7].

Herein, we will report on two hemodialysis patients who suffered from severe Campylobacter infections due to the consumption of raw meat in the same period as the outbreak in Toyama and Kanagawa prefectures. Although they had dined at other restaurant chains and had suffered from other bacterial species, there are several suggestive matters, including the speciality of immune response in hemodialysis patients, and the changes in dietary behaviors and food markets.

\section{Patient 1}

A 56-year-old female bank worker undergoing maintenance hemodialysis complained of a high fever, low back pain, nausea and diarrhea at the beginning of a regular dialysis session. The cause of her end-stage kidney disease (ESKD) was autosomal dominant polycystic kidney disease (ADPKD) and her younger sister also underwent maintenance hemodialysis. She has continued maintenance hemodialysis for 18 years. The patient drank alcohol only on social occasions up to a glass of beer and did not smoke. Five days prior to the onset of symptoms, she attended a farewell party for her colleagues held at a yakitori, i.e. a Japanese-style grilled chicken, restaurant. When her colleague offered her raw chicken sashimi, she had only a small piece because she was afraid of food poisoning. However, the next day, some of the colleagues who joined this party stayed home from work because of diarrhea and fever.

The patient's temperature was $39.6^{\circ} \mathrm{C}$, her pulse was $76 \mathrm{BPM}$ and her systolic/diastolic blood pressure was $148 / 74 \mathrm{~mm} \mathrm{Hg}$. Her body weight during the hemodialysis interval increased by $2 \mathrm{~kg}$ (target dry weight, $49.4 \mathrm{~kg}$ ). The abdomen was generally enlarged and filled with an enlarged cystic liver and kidneys. There was localized resistance with mild tenderness in the right lower quadrant and bowel sounds were hyperactive. There was moderate tenderness over the right costovertebral angle and knocking pain was evident in the area. Clinical laboratory tests revealed leukocytosis with a left shift in the granulocytic series, markedly elevated C-reactive protein (CRP) and fibrinogen (table 1). The CT scan of the abdomen showed hypertrophy of the liver and both kidneys with multiple cysts. Multiple calcification and cysts with higher Hounsfield units than others were also detected (fig. 1).

As soon as a presumptive diagnosis of septicemia and a bacterial gastrointestinal and renal cyst infection was made, empiric treatment with levofloxacin (LVFX) was started. Although the administration of LVFX continued for three days, the fever and low back pain did not change, and leukocytosis and the elevation of CRP continued.

Blood samples from the patient were inoculated into duplicate BACTEC plus Aerobic medium (Becton Dickinson, Sparks, USA) and BACTEC plus+ Anaerobic medium (Becton Dickinson). These bottles were incubated at $35^{\circ} \mathrm{C}$ in the automated blood culture system, BACTEC FX240 (Becton Dickinson), for 5 days. Smears from positive samples from the aerobic bottles were prepared by spreading a drop of the sample over a glass slide and air-drying. The slides were stained using the 
conventional Gram stain procedure and examined. A Campylobacter species was suspected because the smears displayed small, delicate gull wings, D-shaped bacilli, or spirochetal forms. The positive samples were inoculated onto 5\% sheep blood agar (Nissui Pharmaceuticals, Tokyo, Japan) and chocolate agar EX II (Nissui Pharmaceuticals) for the isolation of Campylobacter species and incubated at 35 or $42^{\circ} \mathrm{C}$ for $48 \mathrm{~h}$ in a microaerophilic atmosphere $\left(10 \% \mathrm{CO}_{2}, 5 \% \mathrm{O}_{2}, 85 \% \mathrm{~N}_{2}\right)$. The positive samples were also inoculated on Drigalski agar (Eiken Chemical, Tokyo, Japan) and incubated under an aerobic atmosphere at $35^{\circ} \mathrm{C}$ for $48 \mathrm{~h}$

The bacteria grew and formed smooth and gray colonies in both the 5\% sheep blood agar and chocolate agar under a microaerophilic atmosphere but did not in the Drigalski agar under an aerobic atmosphere. These bacteria grew at both 35 and $42^{\circ} \mathrm{C}$. Sodium hippurate hydrolysis was negative. Antibiotic susceptibility patterns showed resistance to nalidixic acid (30-mg disk) and susceptibility to cephalothin (30-mg disk). Since these phenotypic findings did not allow for a reliable identification, samples were also analyzed with API Campy strips (Biomerieux, Hazelwood, Mo., USA) and API Campy analytical profile index software (Biomerieux). The isolated Gram-negative rods were identified as $C$. upsaliensis and were further confirmed using a molecular method based on 16S rRNA species-specific gene amplification by polymerase chain reaction (PCR) and a subsequent sequence analysis of the PCR product.

An antimicrobial susceptibility test revealed that it was sensitive to ceftriaxone and gentamicin but resistant to LVFX (table 2). The patient was then treated with $2 \mathrm{~g}$ of ceftriaxone and $100 \mathrm{mg}$ of gentamicin instead of LVFX. Eleven days later, the patient recovered and her CRP levels had decreased to the normal range. Following blood and stool cultures were negative for pathogens.

\section{Patient 2}

A 40-year-old male, unemployed, hemodialysis patient, complained of watery diarrhea and a high fever that had persisted for two days at a regular session of hemodialysis. He had undergone maintenance hemodialysis three times per week for 10 years. The cause of his ESKD was chronic hepatitis C-related nephropathy, which had progressed to a Child C liver cirrhosis stage. He was a heavy drinker and took meals out most of the time. One week before the onset, the patient went to an outlet of a yakiniku barbecue restaurant chain, which was different from those that caused the fatal outbreak in Toyama and Kanagawa prefectures, and had eaten yukke, a Korean-style raw beef dish, with alcohol.

His temperature was as high as $39^{\circ} \mathrm{C}$ and his body weight had increased by $2 \mathrm{~kg}$ (target dry weight, $55.4 \mathrm{~kg}$ ), even though he had had an increase of more than $3 \mathrm{~kg}$ between regular intervals of hemodialysis. The systolic/diastolic blood pressure was $140 / 80 \mathrm{~mm} \mathrm{Hg}$ in the right arm and pulse rate was $100 \mathrm{BPM}$. The abdomen was distended, diffusely tender and gurgling sounds were auscultated. Clinical laboratory tests showed an elevated leukocyte count with a left shift in the granulocytic series, a markedly elevated CRP, and moderate abnormalities in liver dysfunction, which is a reflection of endstage liver cirrhosis and azotemia (table 1). Blood samples were also cultured in a BACTEC FX240 system and positive samples were smeared and examined after Gram staining. Since the appearance suggested Campylobacter spp., a selective subculture was performed for patient 1 . The bacteria could not grow at $42^{\circ} \mathrm{C}$. Sodium hippurate hydrolysis was negative. Antibiotic susceptibility patterns showed resistance to nalidixic acid (30-mg disk) and susceptibility to cephalothin (30-mg disk). According to these findings, the patient was diagnosed with bacterial enteritis and septicemia by C. fetus. Simultaneously, the profiles of antibiotics susceptibility for this isolate were examined (table 2).

A treatment of $0.5 \mathrm{~g}$ of meropenem was administered three times to the patient at the end of each hemodialysis session and the patient's fever, abdominal symptoms and diarrhea diminished. Laboratory findings also recovered and following blood cultures became sterile.

\section{Discussion}

Herein, two maintenance hemodialysis patients suffering from Campylobacter infection were reported. The causative microorganism of patient 1 was $C$. upsaliensis 
and that of patient 2 was $C$. fetus. While the most common clinical features of $C$. jejuni and $C$. coli are diarrhea, vomiting and fever in healthy individuals, $C$. upsaliensis and C. fetus lead to more severe complications, especially in patients with underlying diseases $[8,9]$. C. upsaliensis bacteremia is sometimes observed in normal hosts and in opportunistic infections in immunocompromised individuals, and it has been associated with hemolytic uremic syndrome and with postinfectious polyneuropathy $[8,9]$. C. upsaliensis has also been isolated from extraintestinal sites, including a breast abscess and fetoplacental material from a spontaneous human abortion [8]. Patient 1 suffered from ADPKD and it is thought that renal cystitis was brought by $C$. upsaliensis via the blood stream. This is the first reported instance of renal cystitis by $C$. upsaliensis. C. fetus has also been considered as an opportunistic pathogen since most of the affected patients have had an underlying chronic illness, such as liver cirrhosis, diabetes mellitus, cardiovascular diseases and immunodeficiency [10]. C. fetus infections have been reported in severe clinical settings, including bacteremia, septic abortions, meningoencephalitis, motor neuron paralysis, brain abscess, sudural empyema, septic arthritis, vertebral osteomyelitis, lung abscess, peritonitis and cholecystitis $[11,12]$.

Although patients 1 and 2 were undergoing maintenance hemodialysis, the socioeconomic status and lifestyle of each patient was highly contrasted. Patient 1 had a high educational background and her socioeconomic status was also high. She also had a positive attitude toward her life. When she was asked the reason why she had eaten dangerous raw meat dishes during the outbreak of enterohemorrhagic E. coli in Toyama and Kanagawa prefectures, she answered, 'I want to behave as similarly as possible to my healthy colleagues in my bank.' Patient 2 was a heavy drinker and unemployed. He sometimes showed an irresponsible and apathetic attitude. When he was asked the same question, he answered, 'I eat and drink what I want regardless of what might happen to myself.' These responses made us realize the difficulty in guiding the dietary habits according to each personality.

In Japan, Vibrio parahaemolyticus was the most frequent cause of bacterial food poisoning until 1991 and its incidence has since decreased [13]. V. parahaemolyticus mainly contaminates seafood and the Japanese government restricted the sale of raw seafood to below $100 \mathrm{cfu} / \mathrm{g}$ [13]. In contrast, Campylobacter and hemorrhagic E. coli food poisoning have increased since 1997 both because the government had not established a stand for handling poultry meat and because the consumption of poultry meat has greatly increased [14]. Japanese people have traditionally had raw fish as sashimi, but not raw meat. These days, raw foodism, as has come from other ethnicities, is widely accepted [15]. After the food poisoning in Toyama and Kanagawa prefectures in 2011 , the government established a guideline for serving poultry meat, including raw beef and chicken [16].

Both our patients, and the victims in Toyama and Kanagawa prefectures, were served contaminated food in the outlets of the restaurant chains managed by different companies. Oligopolistic markets exist among yakiniku restaurant chains and meat wholesalers in Japan and it was suspected that professional negligence resulted in the outbreak in Toyama and Kanagawa prefectures [17]. Since food poisoning tends to happen simultaneously in multiple and unpredictable areas under these situations [18], hemodialysis patients, as well as healthy people, have a high risk to be victims. 


\section{Conclusion}

Recently, we observed two maintenance hemodialysis patients suffering from food poisoning by Campylobacter. While restriction of potassium, phosphate or water intake has been emphasized in supporting hemodialysis patients, aspects of food sanitation are also important.

\section{Acknowledgements}

We thank the nursing and technical staff of the hemodialysis unit of Juntendo University Hospital for their devoted assistance. We also thank Ms. Ayako Nakamura from the clinical laboratory of Juntendo University Hospital for excellent help with morphologic, biochemical and molecular identification of the Campylobacter species. This work was presented in 2012 at the 57th annual meeting of the Japanese Society for Dialysis Therapy in Sapporo, Japan.

\section{Disclosure Statement}

The authors have nothing to declare.

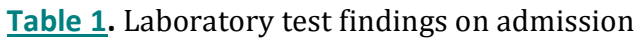

\begin{tabular}{|c|c|c|c|}
\hline & Normal range & Patient 1 & Patient 2 \\
\hline White blood cells, $\mathrm{n} / \mu \mathrm{l}$ & $4,000-8,000$ & 11,000 & 8,800 \\
\hline Neutrophils, \% & $40-70$ & 81.6 & 91.6 \\
\hline Lymphocytes, \% & $20-45$ & 9.1 & 6.6 \\
\hline Monocytes, \% & $4-10$ & 8.9 & 1.8 \\
\hline Eosinophils, \% & $0.5-10$ & 0.3 & 0 \\
\hline Basophils, \% & $0.3-2$ & 0.1 & 0 \\
\hline Hemoglobin, g/dl & $13-17$ & 10.5 & 8.9 \\
\hline Platelets, $\mathrm{n} \times 10^{4} / \mu \mathrm{l}$ & $15-40$ & 21.3 & 5.8 \\
\hline Fibrinogen, mg/dl & $150-400$ & 1,031 & N.D. \\
\hline Aspartate aminotransferase, IU/l & $10-40$ & 12 & 36 \\
\hline Alanine aminotransferase, IU/l & $10-40$ & 8 & 30 \\
\hline Lactate dehydrogenase, IU/l & $115-245$ & 148 & 191 \\
\hline$\gamma$-Glutamyl transpeptidase, IU/l & $<50$ & 13 & 89 \\
\hline Total bilirubin, $\mathrm{mg} / \mathrm{dl}$ & $<1.0$ & 0.42 & 1.11 \\
\hline Blood urea nitrogen, $\mathrm{mg} / \mathrm{dl}$ & $8-20$ & 83 & 23 \\
\hline Creatinine, mg/dl & $0.6-0.9$ & 11.28 & 8.29 \\
\hline Sodium, $\mathrm{mEq} / \mathrm{l}$ & $135-145$ & 130 & 137 \\
\hline Potassium, mEq/l & $3.3-5.1$ & 6.1 & 4.7 \\
\hline Chloride, $\mathrm{mEq} / \mathrm{l}$ & $96-108$ & 91 & 100 \\
\hline C-reactive protein, $\mathrm{mg} / \mathrm{dl}$ & $<0.3$ & 17.9 & 4.1 \\
\hline
\end{tabular}

N.D. = Not done. 
Table 2. The full profiles of antibiotics susceptibility of each isolate

\begin{tabular}{lll}
\hline & $\begin{array}{l}\text { Patient } 1 \\
\text { C. upsaliensis }\end{array}$ & $\begin{array}{l}\text { Patient } 2 \\
\text { C. fetus }\end{array}$ \\
\hline ABPC & $\mathrm{S}$ & $\mathrm{S}$ \\
CEZ & $\mathrm{S}$ & $\mathrm{R}$ \\
CPZ/SBT & $\mathrm{S}$ & $\mathrm{S}$ \\
CTX & $\mathrm{S}$ & $\mathrm{S}$ \\
GM & $\mathrm{S}$ & $\mathrm{S}$ \\
TC & $\mathrm{S}$ & $\mathrm{R}$ \\
EM & $\mathrm{S}$ & $\mathrm{S}$ \\
CLDM & $\mathrm{R}$ & $\mathrm{R}$ \\
NA & $\mathrm{R}$ & $\mathrm{R}$ \\
LVFX & $\mathrm{R}$ & $\mathrm{R}$ \\
\hline
\end{tabular}

$\mathrm{ABPC}=$ Ampicillin; $\mathrm{CEZ}=$ cefazolin $\mathrm{CPZ} / \mathrm{SBT}=$ cefoperazone $/$ sul bactam; CTX = ceftriaxone; $\mathrm{GM}=$ gentamicin; $\mathrm{TC}=$ tetracycline; $\mathrm{EM}=$ erythromycin; $\mathrm{CLDM}=$ clindamycin; $\mathrm{NA}=$ nalidixic acid; $\mathrm{LVFX}=$ levofloxacin; $\mathrm{S}=$ susceptible; $\mathrm{R}$ = resistant.

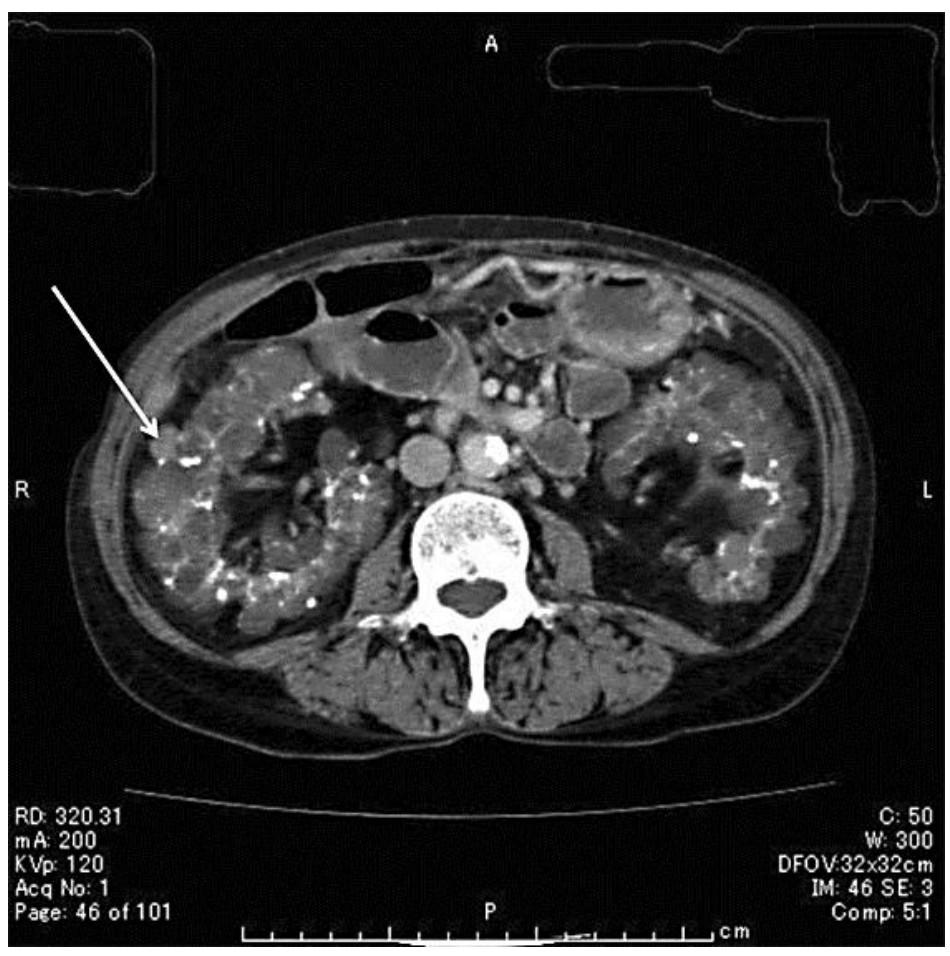

Fig. 1. Enhanced abdominal CT findings of patient 1 (56-year-old woman with ADPKD on maintenance hemodialysis). Both her liver and bilateral kidneys were enlarged and multiple cysts were observed. The arrow indicates a cyst with higher Hounsfield units, which was suspected as being a bacterial cystitis. 


\section{References}

1 Penner JL: The genus Campylobacter: a decade of progress. Clin Microbiol Rev 1988;1:157-172.

2 Skirrow MB, Jones DM, Sutcliffe E, Benjamin J: Campylobacter bacteraemia in England and Wales, 19811991. Epidemiol Infect 1993;110:567-573.

-3 Skirrow MB: Epidemiology of Campylobacter enteritis. Int Food Microbiol 1991;12:9-16.

4 Neimann J, Engberg J, Mølbak K, Wegener HC: A case-control study of risk factors for sporadic campylobacter infections in Denmark. Epidemiol Infect 2003;130:353-366.

5 Lévesque S, Frost E, Arbeit RD, Michaud S: Multilocus sequence typing of Campylobacter jejuni isolates from humans, chickens, raw milk, and environmental water in Quebec, Canada. J Clin Microbiol 2008;46:3404-3411.

6 Matano S, Inamura K, Konishi M, et al: Encephalopathy, disseminated intravascular coagulation, and hemolytic-uremic syndrome after infection with enterohemorrhagic Escherichia coli 0111. J Infect Chemother 2011. [Epub ahead of print]

7 Restaurants, retailers banned from providing raw beef liver. Manichi Japan (July 1, 2012). http://mainichi.jp/english/english/newsselect/news/20120701p2g00m0bu003000c.html.

8 Bourke B, Chan VL, Sherman P: Campylobacter upsaliensis: waiting in the wings. Clin Microbiol Rev 1998;11:440-449.

$\$ 9$ Fernández-Cruz A, Muñoz P, Mohedano R, et al: Campylobacter bacteremia: clinical characteristics, incidence, and outcome over 23 years. Medicine (Baltimore) 2010;89:319-330.

10 Monno R, Rendina M, Ceci G, et al: Campylobacter fetus bacteremia in an immunocompromised patient: case report and review of the literature. New Microbiol 2004;27:281-285.

11 Sauerwein RW, Bisseling J, Horrevorts AM: Septic abortion associated with Campylobacter fetus subspecies fetus infection: case report and review of the literature. Infection 1993;21:331-333.

12 Wheeler AP, Gregg CR: Campylobacter bacteremia, cholecystitis, and the acquired immunodeficiency syndrome. Ann Intern Med 1986;105:804.

13 WHO: Vibrio parahaemolyticus, Japan, 1996-1998. Wkly Epidemiol Rec 1999;74:361-363.

14 Obana M, Sagara H, Aoki T, et al: The current status of infectious enteritis in Japan - reports of the 'Research Group for Infectious Enteric Diseases, Japan' in the last 5 years (1996-2000). Kansenshogaku Zasshi 2002;76:355-368. Japanese

15 Kamiya M, Ooi HK: Current status of food-borne parasitic zoonoses in Japan. Southeast Asian J Trop Med Public Health 1991;22(suppl):48-53.

16 E. coli detected in cow livers/Ministry likely to tighten controls on serving meat uncooked. Daily Yomiuri Online (December 16, 2011). http://www.yomiuri.co.jp/dy/national/T111215005067.htm.

17 Death toll in food poisoning at 'yakiniku' chain reaches 4. The Japan Times (May 6, 2011). http://search.japantimes.co.jp/cgi-bin/nn20110506a3.html. 